\title{
THE RED SUPERGIANT CONTENT OF M31*
}

\author{
Philip Massey ${ }^{1,2}$ and Kate Anne Evans ${ }^{1,3}$ \\ ${ }^{1}$ Lowell Observatory, 1400 W Mars Hill Road, Flagstaff, AZ 86001-4499, USA; kevans@caltech.edu, phil.massey@lowell.edu \\ ${ }^{2}$ Department of Physics and Astronomy, Northern Arizona University, Flagstaff, AZ 86011-6010, USA \\ Received 2016 April 16; revised 2016 May 24; accepted 2016 May 25; published 2016 August 2
}

\begin{abstract}
We investigate the red supergiant (RSG) population of M31, obtaining the radial velocities of 255 stars. These data substantiate membership of our photometrically selected sample, demonstrating that Galactic foreground stars and extragalactic RSGs can be distinguished on the basis of $B-V, V-R$ two-color diagrams. In addition, we use these spectra to measure effective temperatures and assign spectral types, deriving physical properties for 192 RSGs. Comparison with the solar metallicity Geneva evolutionary tracks indicates astonishingly good agreement. The most luminous RSGs in M31 are likely evolved from 25-30 $M_{\odot}$ stars, while the vast majority evolved from stars with initial masses of $20 M_{\odot}$ or less. There is an interesting bifurcation in the distribution of RSGs with effective temperatures that increases with higher luminosities, with one sequence consisting of early K-type supergiants, and with the other consisting of M-type supergiants that become later (cooler) with increasing luminosities. This separation is only partially reflected in the evolutionary tracks, although that might be due to the mis-match in metallicities between the solar Geneva models and the higher-than-solar metallicity of M31. As the luminosities increase the median spectral type also increases; i.e., the higher mass RSGs spend more time at cooler temperatures than do those of lower luminosities, a result which is new to this study. Finally we discuss what would be needed observationally to successfully build a luminosity function that could be used to constrain the mass-loss rates of RSGs as our Geneva colleagues have suggested.
\end{abstract}

Key words: galaxies: individual (M31) - galaxies: stellar content - Local Group - stars: massive - supergiants

Supporting material: machine-readable tables

\section{INTRODUCTION}

Massive stars spend most of their lives as OB stars. The most massive of these $\left(>\sim 40 M_{\odot}\right)$ then evolve into Wolf-Rayet (WR) stars, possibly after passing through a luminous blue variable (LBV) phase. In contrast, massive stars with masses below $\sim 30 M_{\odot}$ spend their He-burning lives as red supergiants (RSGs) after passing through a yellow supergiant (YSG) stage during their quick journey across the Hertzsprung-Russell diagram (HRD). At some intermediate masses $\left(\sim 30 M_{\odot}\right.$, say) stars may pass through both a RSG and WR phase, passing through the YSG phase twice. The exact mass ranges corresponding to these various stages, along with the relative lifetimes spent in these regions of the HRD, depend heavily on the initial metallicity of the gas out of which these stars form, as radiation pressure acting on highly ionized metals results in mass loss that strongly influences this evolution. Thus, characterizing the luminous populations of nearby galaxies allows us to perform exacting tests of massive star evolution as a function of metallicity. (For a recent review, see Massey 2013.)

The Andromeda Galaxy (M31) plays a unique role in such studies, as it provides the only nearby extragalactic example where the metallicity is solar and above (Zaritsky et al. 1994; Sanders et al. 2012). Previous work on the massive star population of M31 has established the WR content (Neugent et al. 2012a), identified YSGs (Drout et al. 2009), discovered

\footnotetext{
* Observations reported here were obtained at the MMT Observatory, a joint facility of the University of Arizona and the Smithsonian Institution. This paper uses data products produced by the OIR Telescope Data Center, supported by the Smithsonian Astrophysical Observatory.

${ }^{3}$ Research Experience for Undergraduate participant during the summer of 2015. Current address: California Institute of Technology, 1200 East California Blvd, Pasadena, CA 91125.
}

LBV candidates (Massey 2006; Massey et al. 2007a, 2016), and laid the groundwork for the current study by performing a preliminary reconnaissance of its RSG population (Massey 1998; Massey et al. 2009). Work on the unevolved massive star population suggests that only a few percent of the total number $\mathrm{O}$ stars and $\mathrm{B}$ supergiants have been identified (Massey et al. 2016).

In this paper we set out to complete our identification of the RSG population of M31 down to $\sim 15 M_{\odot}$, using a combination of spectroscopy and photometry. Massey et al. (2007b) showed that samples of red stars in the right color and magnitude range to be RSGs were badly contaminated $(\sim 80 \%)$ by foreground red dwarfs. However, for cool stars $\left(T_{\text {eff }} \leqslant 4300 \mathrm{~K}\right), B-V$ becomes primarily an indicator of surface gravity due to lineblanketing in the $B$ band, while $V-R$ remains primarily a temperature indicator (Massey 1998). Thus there is a relatively clean separation in a $B-V$ versus $V-R$ two-color plot between foreground dwarfs and RSGs. We show an example of such an effort in Figure 1 based upon Massey et al. (2009).

Prior efforts at spectroscopic confirmation have been relatively modest. Massey (1998) "confirmed" about 20 RSGs in M31. In some cases the spectroscopic confirmation is based on clear radial velocity information, but in other cases it is based on softer criteria, such as the strengths of the $\mathrm{Ca}$ II triplet lines; these criteria were not always in good agreement. Massey et al. (2009) identified a sample of 437 RSG candidates in M31 from the Local Group Galaxy Survey (LGGS) $B V R$ photometry of Massey et al. (2006), and obtained radial velocities of 124 of these stars, but derived physical properties (effective temperatures and bolometric luminosities) for only 16 stars in their sample. Furthermore, the photometrically selected RSG and foreground candidates did not always prove to be what was 


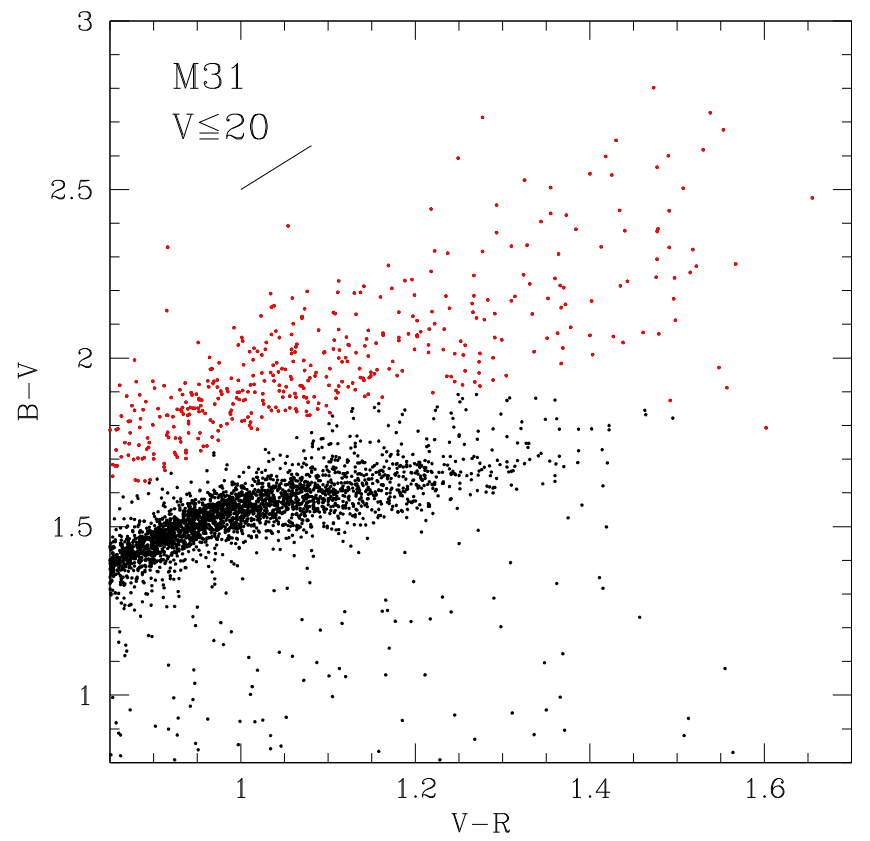

Figure 1. Two-color diagram of red stars in M31. The points in red are suspected RSGs from Table 1 of Massey et al. (2009), while the points in black represent suspected foreground stars. The photometry is taken from the LGGS (Massey et al. 2006), with $V \leqslant 20.0$. The typical reddening vector for M31 stars is shown by the diagonal line.

expected when observed spectroscopically. We decided it was time for a more complete study.

This investigation is timely. As the evolutionary models improve, knowledge of the RSG content of M31 will provide us with a powerful magnifying glass ${ }^{4}$ for examining the predictions of these models, and for better constraining the input. For instance, Maeder et al. (1980) argued that the relative number of RSGs and WRs should be a strong function of the metallicity, with proportionately fewer RSGs found at higher metallicities. Further, the number of RSGs gives another way of estimating the expected number of unevolved $\mathrm{O}$ stars, a value that is poorly constrained observationally (Massey et al. 2016).

An even more exciting possibility is for us to use our knowledge of the RSG content of M31 to determine the massloss rates for RSGs. As recently shown by Meynet et al. (2015) the time-averaged mass-loss rates of RSGs are very poorly constrained by observations, as much of the action occurs episodically. The consequences of this significantly affect our interpretation of the populations of the upper part of the HRD. For instance, according to the models, enhanced mass loss during the RSG phase leads to the prediction that the majority of blue and YSGs are post-RSG objects (Meynet et al. 2015). In addition, enhanced mass-loss rates during the RSG phase may solve the so-called "red supergiant problem." RSGs have long been thought to be the progenitors of type II-P supernovae; Smartt et al. (2009), however, argued that the observed upper mass limit of the progenitors of type II-P supernovae is 17-18 $M_{\odot}$ rather than the $30 M_{\odot}$ or more one would expect from what the models. Ekström et al. (2012) argues that an improved mass-

\footnotetext{
4 Kippenhahn \& Weigert (1990 p. 192) liken these evolved stages to "a sort of magnifying glass, [which reveals] relentlessly the faults of calculations of earlier phases." We are indebted to our colleague Andre Maeder for calling this quote to our attention.
}

loss prescription during the RSG phase leads naturally to this result, as the mass-loss rates are then significantly enhanced for the highest mass RSGs, leading to a likely scenario where these stars lose most of their hydrogen-rich envelopes and exploded instead as type II-L or even type Ib supernovae.

The validity of this resolution rests on the assumption that the RSG mass-loss rates have been seriously underestimated. Meynet et al. (2015) and Georgy et al. (2015) proposed a novel way of determining if this is correct. The evolution models predict that the luminosity functions of RSGs will have a strong dependence on the average mass-loss rates for RSGs, becoming steeper (fewer high luminosity RSGs) with higher mass-loss rates. However, this requires an unbiased knowledge of the RSG content of a mixed-age population in which the (global) star-formation rate has remained essentially constant over the relevant evolution time period (20-30 Myr). Model calculations are currently underway for this undertaking. Our study here does not provide an answer, but it provides an vital toe-inthe-door that delineates the observational hurdles associated with constructing the required bias-free luminosity function.

In Section 2 we will explain our sample selection and the spectroscopy we obtained for our study. In Section 3 we will use these data for our analysis, first distinguishing RSGs from foreground stars using the radial velocities (Section 3.1) and comparing these results to our expectations based on our twocolor diagrams. We will also measure effective temperatures and spectral types (Section 3.2), and compute other physical properties, such as the bolometric luminosities. We will then compare these to what the Geneva evolutionary tracks predict (Section 3.3). Finally, in Section 4 we will summarize our results and discuss what future work is needed in order to construct a sufficiently deep luminosity function for constraining the RSG mass-loss rates.

\section{OBSERVATIONS}

\subsection{Sample Selection}

Our primary goal was to see how accurately we could use the $(V-R, B-V)$ two-color selection method of Massey (1998) to distinguish actual RSGs from Galactic foreground stars. In this way, M31 serves as an excellent (high-metallicity) test bench. For some galaxies, such as the Magellanic Clouds, most of the systemic radial velocity is actually due to the reflex motion of the Sun. Thus, although foreground disk dwarfs are easily distinguished from Magellanic Cloud members (Neugent et al. 2010, 2012b), halo red giants would be hard to distinguish from Magellanic Cloud RSGs on the basis of radial velocities alone. (Statistically, however, we can argue that few halo red giants are expected in the appropriate magnitude range; see Section 4 of Neugent et al. 2012b.) M31 has a systemic velocity of $\sim-300 \mathrm{~km} \mathrm{~s}^{-1}$ and a rotational velocity of $\sim 250 \mathrm{~km} \mathrm{~s}^{-1}$ (Massey et al. 2009 and references therein.) Thus there is an "alligator's jaws" shaped area in the NE section of M31 where it is difficult to separate foreground dwarfs from M31 members, but over most of the galaxy the separation is very clean. (See Figure 5 of Drout et al. 2009.)

In selecting targets to observe, we used the 437 photometrically selected RSG candidates given in Table 1 of Massey et al. (2009). These are the stars marked as red in Figure 1 and they meet the following criteria: (1) $V<20$, corresponding roughly to $\log L / L_{\odot} \sim 4.4$ for early K-type stars and $\log L / L_{\odot} \sim 4.8$ for mid M-type stars. (2) $V-R \geqslant 0.85$ (and correspondingly 
$B-V \geqslant 1.5)$ to restrict the sample to K-type stars and later, roughly corresponding to $T_{\text {eff }}<4300 \mathrm{~K}$ given typical reddening for M31 RSGs. ${ }^{5}$ (3) $B-V>-1.599(V-R)^{2}+$ $4.18(V-R)-0.83$ to separate the low surface gravity RSGs from the high surface gravity foreground Galactic stars. Some of these stars had previously measured radial velocities (Table 2 of Massey et al. 2009), which could then serve as radial velocity templates. Of the 437 stars, $36(8.2 \%)$ of them were somewhat crowded and given lower priorities in the Hectospec assignments.

This project was "piggy-backed" on our primary program to continue to monitor a sample of WR binaries in M31 (Neugent \& Massey 2014). We observed six of these WR configurations with slightly different supergiant and foreground candidate red stars included on the otherwise unused fibers. In addition, we observed one configuration that consisted only of red stars. In all we successfully observed 255 RSG candidates and 98 foreground candidates, many of them multiple times.

\subsection{Spectroscopy}

The observations were all made with Hectospec, a 300-fiber spectrometer on the $6.5 \mathrm{~m}$ MMT (Fabricant et al. 2005). The 270 lines $\mathrm{mm}^{-1}$ grating was used, providing a reciprocal dispersion of $1.2 \AA$ pixel $^{-1}$, and a spectral resolution of 4.5-5.2 $\AA$ from 3650-9200 $\AA$ in first order. Since no blocking filter is used, there is some contamination from second-order blue in the far red, but given the colors of these stars, this is pretty minimal; the grating is blazed at $5000 \mathrm{~A}$. The six "WR configurations" were observed for 90 minutes each (UT 2014 September 24, September 26, November 21, November 22, November 26, and November 28); the one "red star" configuration was observed for 60 minutes (UT 2014 October 1). The observations were made in a selfstaffed queue; P. M. and collaborator Kathryn Neugent were present for the November observing.

The reductions were previously described by Evans \& Massey (2015). For each of the seven configurations, some fibers were assigned to blank sky in order to facilitate sky subtraction. Calibration exposures included $\mathrm{HeNeAr}$ and quartz lamp exposures. The data were all run through the SAO pipeline, with the night-sky lines used to adjust the wavelength zero-points relative to the HeNeAr exposures that were made in the afternoon. As part of the reduction procedure, the wavelengths were corrected to the heliocentric rest frame. Observations of the spectrophotometric standard Feige 34 were made midway through the semester, and were used to produce sensitivity curves by Nelson Caldwell, who kindly made these available to us.

\section{ANALYSIS}

\subsection{Membership: RSGs versus Foreground Stars}

We measured radial velocities from each of the spectra using $\mathrm{XCSAO}$, an IRAF ${ }^{6}$ cross-correlation tool. As described in Evans

\footnotetext{
5 Our interpretation of the $V-R$ cutoff requirements differs slightly from that stated by Massey et al. (2009), who state that $V-R \geqslant 0.85$ corresponds to an intrinsic $(V-R)_{0}$ of $0.81 \quad\left(T_{\text {eff }} \leqslant 4000 \mathrm{~K}\right)$. Yet, we expect $E(V-R) \sim 0.6 E(B-V)$ (Schlegel et al. 1998). The M31 OB stars in the LGGS have a median reddening of $E(B-V)=0.13$, so this would correspond to $E(V-R)_{0}=0.77$, not 0.81 . But, the actual M31 RSG sample has higher reddening, with $E(B-V) \sim 0.3$ for reasons probably associated with circumstellar dust (Massey et al. 2005). Thus, $E(V-R) \sim 0.18$ and $(V-R)_{0} \sim 0.67$, corresponding more to an $T_{\text {eff }} \leqslant 4300 \mathrm{~K}$. This allows earlytype $\mathrm{K}$ stars to be included in the sample.

6 IRAF is distributed by the National Optical Astronomy Observatory, which is operated by the Association of Universities for Research in Astronomy (AURA) under a cooperative agreement with the National Science Foundation.
}

\& Massey (2015), we used 21 Hectospec spectra of six M31 RSGs for which Massey et al. (2009) had already determined radial velocities. (Those velocities, in turn, were tied to three legitimate late-type radial velocity standards.) The crosscorrelation was restricted to the $8350-8750 \AA$ region around the Ca II $\lambda \lambda 8498,8542,8662$ triplet, lines that are very strong in RSGs. This process also allows us to avoid the wide molecular features, atmospheric bands, and so on. For each individual program spectrum, a single velocity was produced by averaging the results from the 21 velocity templates, weighting each appropriately by the internal error from the cross-correlation (i.e., weights were the squares of the reciprocal errors). For the stars with multiple observations, a weighted average was then determined. ${ }^{7}$ We list the radial velocities in Table 1.

Massey et al. (2009) demonstrated that the radial velocities of M31's H II regions and RSGs agreed very well, and that the expected radial velocities of these Population I objects could be approximated by a linear relationship with $(X / R)$,

$$
V_{r}=-295+241.5(X / R),
$$

where $X$ is the distance along the semimajor axis and $R$ is the galactocentric distance within the plane of M31. Such a linear approximation agrees well with the more complex twodimension velocity field determination of Sofue \& Kato (1981) and other studies (e.g., Hurley-Keller et al. 2004), and is equivalent to saying that the rotation curve is flat. In contrast, we expect the foreground red stars to cluster around a velocity of $0 \mathrm{~km} \mathrm{~s}^{-1}$, with some scatter indicative of the radial velocity dispersion of nearby red dwarfs in this particular direction.

In Figure 2 we show a plot of the radial velocity with $(X / R)$ for all of our data. We see that the vast majority of the candidate RSGs (red points) follow the expected relationship between $V_{r}$ and $(X / R)$, while the vast majority of the candidate foreground stars (black points) cluster around $0 \mathrm{~km} \mathrm{~s}^{-1}$ as expected. The foreground candidates were observed only as part of the single "red star" configuration, and hence are distributed over the limited range of $(X / R) \sim 0.25-1.0$. We have used the larger sample size here to improve on the radial velocity relation, deriving

$$
V_{r}=-311.8+242.0(X / R) .
$$

Over the range $(X / R)=-1$ to +1 , the expected $V_{r}$ from this relationship differs from that of the Massey et al. (2009) determination by -16.3 to $-17.3 \mathrm{~km} \mathrm{~s}^{-1}$. This may be compared to the overall scatter around the relationship of 25 $\mathrm{km}^{-1}$. Recall that the radial velocity "standards" for the new measurements came from adopting the radial velocities determined by Massey et al. (2009). We checked our new measurements against the old to make sure this $\sim-17 \mathrm{~km} \mathrm{~s}^{-1}$ was due to better coverage in $(X / R)$ and not some reduction issue. Indeed, there is only a small systematic offset between the new and old measurements; the mean difference (in the sense of new minus old) is $-7.4 \mathrm{~km} \mathrm{~s}^{-1}$ with a scatter of 12.3 $\mathrm{km} \mathrm{s}^{-1}$ and median difference $-5.9 \mathrm{~km} \mathrm{~s}^{-1}$ using 73 stars in

\footnotetext{
7 Prior to 2014, the SAO pipeline reductions did not include correcting the wavelength scale to heliocentric; this change caused a certain amount of confusion and consternation during our initial attempts to understand our results. We are indebted to Nelson Caldwell for help in tracking down this issue.
} 
Table 1

Radial Velocities of RSG and Foreground Candidates

\begin{tabular}{|c|c|c|c|c|c|c|c|c|}
\hline Star & 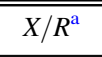 & $\overline{V_{\mathrm{M} 31}{ }^{\mathrm{b}}}$ & $\bar{V}_{\mathrm{obs}}^{\mathrm{b}}$ & $\bar{V}_{\mathrm{err}}^{\mathrm{b}}$ & $N_{\text {obs }}$ & $\overline{\text { TDR }^{\mathrm{c}}}$ & Old $V_{\text {obs }}^{\text {b,d }}$ & Phot. Type \\
\hline J003950.86+405332.0 & -0.586 & -436.5 & -433.0 & 0.5 & 6 & 14.4 & $\cdots$ & RSG \\
\hline J003950.98+405422.5 & -0.563 & -431.0 & -418.4 & 0.7 & 3 & 14.5 & $\cdots$ & RSG \\
\hline J003957.00+410114.6 & -0.409 & -393.8 & -446.9 & 0.6 & 6 & 12.7 & -442.4 & RSG \\
\hline J004015.86+405514.1 & -0.638 & -449.0 & -387.9 & 0.9 & 4 & 8.7 & $\cdots$ & RSG \\
\hline J004019.15+404150.8 & -0.999 & -536.2 & -564.6 & 1.2 & 1 & 13.4 & -551.9 & RSG \\
\hline J004020.06+410651.3 & -0.326 & -373.8 & -362.6 & 0.5 & 5 & 15.8 & $\cdots$ & RSG \\
\hline
\end{tabular}

Notes.

${ }^{\mathrm{a}} X$ is the distance along the semimajor axis and $R$ is the galactocentric radius within the plane of M31.

${ }^{\mathrm{b}}$ Radial velocities in $\mathrm{km} \mathrm{s}^{-1}$.

c Tonry \& Davis (1979) $r$-parameter.

d From Massey et al. (2009).

e Photometric type based upon $B-V$ versus $V-R$; fgd-foreground.

(This table is available in its entirety in machine-readable form.)

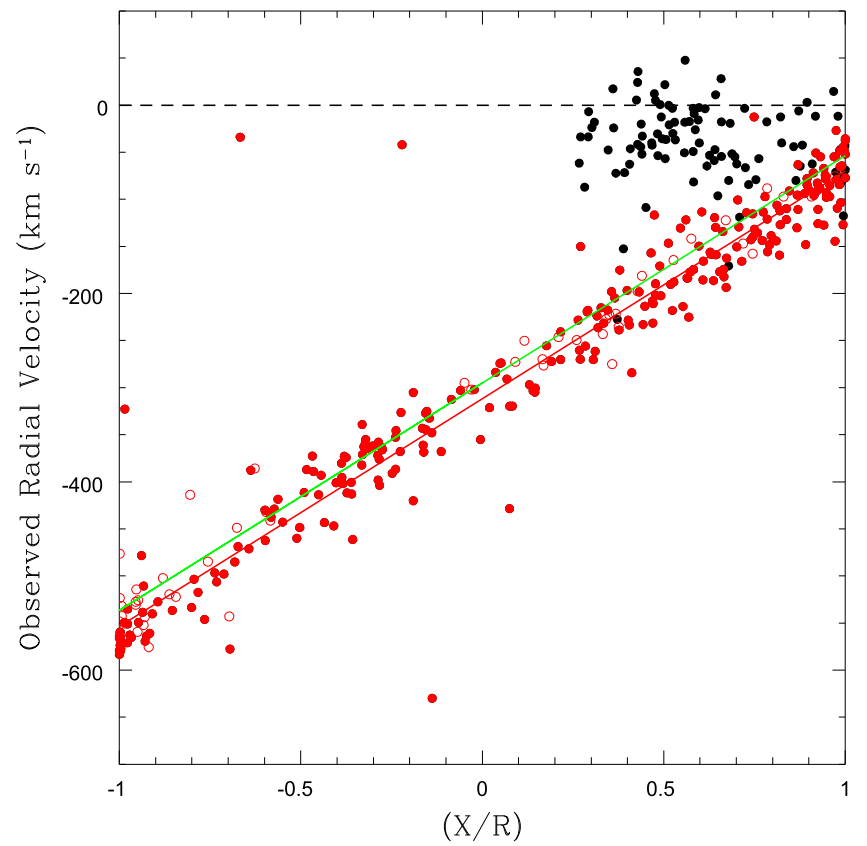

Figure 2. Radial velocities of foreground and RSG candidates. The radial velocities of our foreground (black) and RSG (red) candidates are plotted against $(X / R)$, where $X$ is the distance along the semimajor axis, and $R$ is the galactocentric separation within the plane of M31. Filled red circles are from the present paper; open red circles are from Massey et al. (2009). The black dashed line indicates $0 \mathrm{~km} \mathrm{~s}^{-1}$ and we expect that foreground galactic stars will cluster near this value. The green line denotes the radial velocity vs. $(X / R)$ relation for M31 from Massey et al. (2009), while the red line denotes the revised version given here.

common. $^{8}$ If we include the 50 additional RSGs with radial velocities from Massey et al. (2009) for which we do not have new radial velocities, we obtain a very similar relation,

$$
V_{r}=-310.0+239.7(X / R) \text {. }
$$

\footnotetext{
8 This excludes one outlier with a huge difference, J004217.99+410912.7, whose velocity measurement here is $118 \mathrm{~km} \mathrm{~s}^{-1}$ more positive than that in Massey et al. (2009). Neither measurement agreed well with the expected radial velocity; possibly the star is a radial velocity variable (binary).
}

Although our photometric classification was obviously extremely successful, we can see that there were a few RSGs misclassified photometrically as foreground stars, a few foreground stars misclassified as RSGs, and a few RSGs with interestingly discrepant radial velocities. Let us consider each of these cases in turn.

Drout et al. (2009) argued that confusion with foreground stars sets in at radial velocities $\geqslant-150 \mathrm{~km} \mathrm{~s}^{-1}$. There are two foreground candidates (black points) more negative than that in Figure 2, each near the RSG velocity relation. In other words, two stars whose velocities indicate they are indeed RSGs despite having been classified photometrically as foreground stars. These are J004458.49+421219.2 and J004406.92 +412307.2 . We denote their position in the two-color diagram in Figure 3 with large green points. We see that one of these stars (J004458.49+421219.2) has photometry right on the border between what we defined as a RSG candidate and what we called a foreground candidate; the other, J004406.92 +412307.2 , really "should" be a foreground star according to the photometry.

There are three stars photometrically classified as RSGs that have velocities consistent with their actually being foreground stars: J004105.97+403407.9, J004303.26+404710.9, and J004431.71+415629.1. We mark two of these stars with large magenta symbols in Figure 3; the third (J004105.97 $+403407.9)$ has such unrealistic photometry $(V-R \sim 4.7)$ that it would fall well to the right on the plot. That star is extremely crowded according to the LGGS. The other two stars have photometry that is right on the borderline between the separation of RSGs and foreground stars. Of these, the one with the redder $V-R$ has very poor spectra, as well as a surprisingly large $V-R$ color.

Thus, of all 354 stars with new data, only one star is surprisingly inconsistent with its photometric classification versus its radial velocity. We conclude that the photometric classification using the $V-R, B-V$ two-color diagram appears to be a very robust way of separating foreground stars from RSGs without needing radial velocities, at least at high metallicities.

The final type of discrepancy is when a RSG candidate has a surprising velocity with respect to its position in M31, but its 


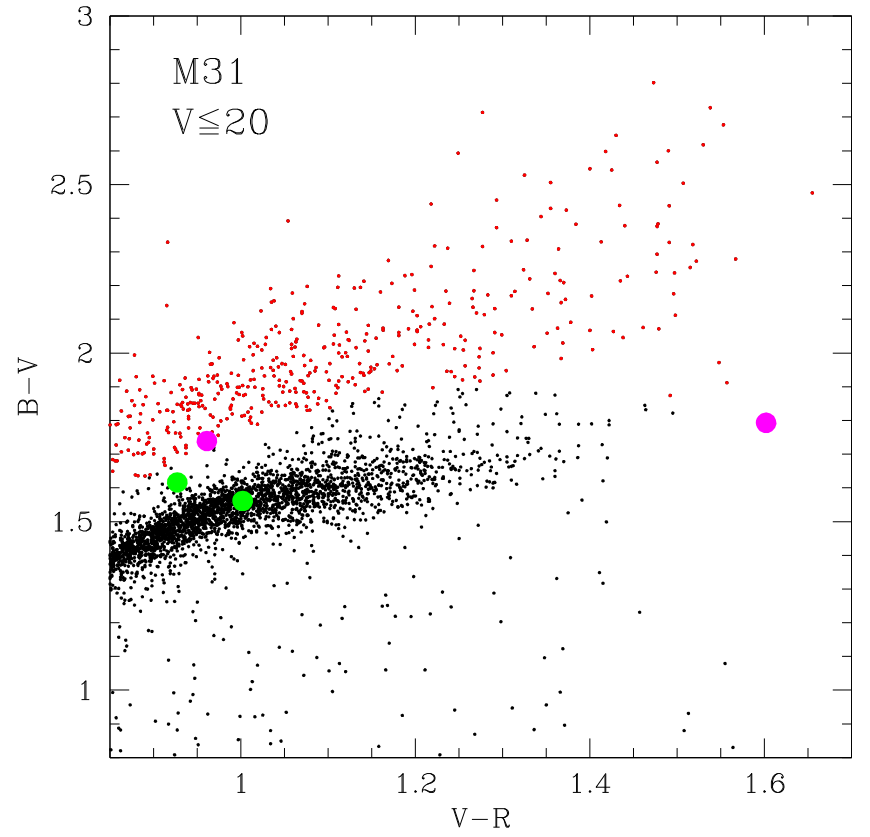

Figure 3. Location of discrepantly classified stars (from Figure 2) in two-color diagram. The same as Figure 1 but with large symbols denoting the location of the four stars whose radial velocities disagree with the photometric classification. Green symbols are stars photometrically classified as foreground objects but whose radial velocities are consistent with M31 membership, while magenta symbols are stars photometrically classified as RSGs, but whose radial velocities are consistent with membership in the Milky Way. A fifth star, whose photometry is confused by crowding, is located off the right side of this plot.

velocity is not consistent with it being a foreground star either. Evans \& Massey (2015) discuss J004330.06+405258.4, the star with a radial velocity of $-630 \mathrm{~km} \mathrm{~s}^{-1}$ at $(X / Y)=-0.138$. They conclude that this is an bona fide runaway RSG, the first discovered on the basis of its radial velocity, and the first known extragalactic massive star runaway. A significant percentage $(10 \%-50 \%)$ of unevolved massive stars are OB runaways (Gies \& Bolton 1986, and references therein), with discrepant radial velocities $\left(>30 \mathrm{~km} \mathrm{~s}^{-1}\right)$ compared to other OB stars in their neighborhood. Runaways probably occur via dynamical evolution (Gies \& Bolton 1986; Fujii \& Portegies Zwart 2011). Few evolved massive runaways are known, doubtless because of the difficulties in recognizing such objects once they are older and have moved further from their birthplace. J004330.06+405258.4 has a radial velocity that is $300 \mathrm{~km} \mathrm{~s}^{-1}$ more negative than that expected from M31's rotation curve, and Evans \& Massey (2015) note that the object is located some $\sim 20^{\prime}$ (4.6 kpc) from the plane of the disk, consistent with it having a transverse velocity similar to its radial velocity motion.

We find three other potential runaways in Figure 2: $\mathrm{J} 004217.99+410912.7$ has a radial velocity of $-323 \mathrm{~km} \mathrm{~s}^{-1}$ rather than the $-533 \mathrm{~km} \mathrm{~s}^{-1}$ expected for $(X / R)=-0.985$. We have five observations of this star, all with similar velocities. However, an older measurement by Massey et al. (2009) disagrees with these, with a value of $-440 \mathrm{~km} \mathrm{~s}^{-1}$. The difference in radial velocity between the Fall 2014 observations reported here and the Fall 2005 observations of Massey et al. (2009) might suggest the star is a long-period binary, but the large velocity difference would be more indicative of a shortperiod binary, which we can essentially rule out from our five new measurements. These results are puzzling.
$\mathrm{J} 004112.27+403835.8$ and $\mathrm{J} 004228.99+412029.7$ can be seen in Figure 2 at $\left(-0.695,-577 \mathrm{~km} \mathrm{~s}^{-1}\right)$ and $(0.075,-428$ $\left.\mathrm{km} \mathrm{s}^{-1}\right)$. These stars also might be runaways as their radial velocities are expected to be $-462 \mathrm{~km} \mathrm{~s}^{-1}$ and $-277 \mathrm{~km} \mathrm{~s}^{-1}$, respectively. Both stars have multiple observations, with good agreement in their measurements, as evidenced by the low standard errors of the means given in Table 1, i.e., 1.0 and 1.3 $\mathrm{km} \mathrm{s}^{-1}$. However, the spectrum of J004228.99+412029.7 is that of a very early K-type, or even late G-type; this is consistent with the low value for the Tonry \& Davis (1979) $r$ parameter we obtained, indicating a relatively poor match with the M-type radial velocity templates we used in measuring the velocities.

\subsection{Effective Temperatures, Spectral Types, and Physical Parameters}

Spectral types for the M31 RSGs were determined by comparing their spectra to those of Galactic RSGs observed by Levesque et al. (2005). Those Galactic RSGs had spectral types that were generally well established in the literature, although in a few cases they were reclassified by Levesque et al. (2005). The agreement between these were usually exact, although occasionally there would be a difference of one one spectral subtype, i.e., M1 I versus M2 I. The primary criteria were those described by Levesque et al. (2005), i.e., the strengths of the $\mathrm{TiO}$ bands for M- and late K-types, and the strengths of the $\mathrm{G}$ band and $\mathrm{Ca}$ I $\lambda$ 4226. We list these spectral types in Table 2. In the few cases where no spectral type could be assigned, the stars are likely not RSGs at all, but are somewhat earlier members of M31.

Effective temperatures were determined following the procedures described by Levesque et al. (2005) using the $2 \times$ solar metallicity MARCS atmosphere models used by Massey et al. (2009). For the vast majority of our M31 RSGs we had multiple observations; each of these spectra were fit independently; what are shown are the median values. The uncertainty in our fitting is approximate $50 \mathrm{~K}$. These temperatures are given in Table 2. Note that in a few cases effective temperatures could not be measured reliability.

There are eight stars in common between our study here and that of Massey et al. (2009); we list a comparison of the spectral types and effective temperatures in Table 3 . In preparing this we were chagrined to discover a significant error in Massey et al. (2009): the effective temperatures they listed were based upon the solar metallicity MARCS models and not the $2 \times$ solar metallicity MARCS model as stated in the text. Experimentation showed that this introduced a systematic difference of $75 \mathrm{~K}$, i.e., that fitting the spectra used by Massey et al. (2009) with the $2 \times$ solar model rather than the $1 \times$ solar model require temperatures that are roughly $75 \mathrm{~K}$ higher. We have therefore adjusted the Massey et al. (2009) effective temperatures by that amount in making this comparison. We find the agreement in the effective temperatures determined from our new spectra to be quite consistent. The only significant discrepancy in spectral type is for J003957.00 +410114.6 . We have six observations of that star; all yield a spectral type in the range of M3-M4 I, consistent with our effective temperature. We are forced to conclude that the Massey et al. (2009) spectral type is a mistake rather than a sign of variability, given that the effective temperature given for the star by Massey et al. (2009) is much cooler than what they found for other M0 I stars, and is more like that of a M3 I type. 
Table 2

Physical Properties

\begin{tabular}{|c|c|c|c|c|c|c|c|}
\hline \multirow[b]{2}{*}{ Star } & \multirow[b]{2}{*}{ Type } & \multirow[b]{2}{*}{$T_{\text {eff }}$} & \multicolumn{2}{|c|}{$K$-band Photom. } & \multirow[b]{2}{*}{$M_{K}$} & \multirow[b]{2}{*}{$\log L / L_{\odot}$} & \multirow[b]{2}{*}{$R / R_{\odot}$} \\
\hline & & & $K_{s}$ & Source $^{\mathrm{a}}$ & & & \\
\hline $\mathrm{J} 003950.86+405332.0$ & M1 I & 3850 & 14.45 & M & -10.03 & 4.86 & 600 \\
\hline $\mathrm{J} 003950.98+405422.5$ & M4 I & 3650 & 14.20 & M & -10.28 & 4.89 & 700 \\
\hline $\mathrm{J} 003957.00+410114.6$ & M4 I & 3650 & 15.08 & $\mathrm{~F}$ & -9.40 & 4.54 & 470 \\
\hline J004015.18+405947.7 & M3 I & 3700 & 14.26 & M & -10.22 & 4.89 & 670 \\
\hline $\mathrm{J} 004015.86+405514.1$ & $\mathrm{~K} 2 \mathrm{I}$ & 3950 & 15.62 & M & -8.86 & 4.42 & 340 \\
\hline $\mathrm{J} 004019.15+404150.8$ & M2 I & 3750 & 14.61 & M & -9.87 & 4.76 & 570 \\
\hline $\mathrm{J} 004020.06+410651.3$ & M0 I & 3900 & 14.60 & M & -9.88 & 4.81 & 560 \\
\hline J004023.84+410458.6 & K5 I & 3975 & 15.69: & M & -8.79 & 4.40 & 330 \\
\hline $\mathrm{J} 004025.36+404623.1$ & M0 I & 3900 & 15.29: & M & -9.19 & 4.53 & 400 \\
\hline $\mathrm{J} 004025.75+404254.8$ & K5 I & 3925 & $\cdots$ & $X$ & $\cdots$ & $\cdots$ & \\
\hline
\end{tabular}

Note.

${ }^{a}$ M-2MASS (Cutri et al. 2003), F-FLAMINGOS (Massey et al. 2009), X-no data.

(This table is available in its entirety in machine-readable form.)

Table 3

Comparison with Massey et al. (2009)

\begin{tabular}{|c|c|c|c|c|}
\hline \multirow{2}{*}{ Star } & \multicolumn{2}{|c|}{ Spectral Type } & \multicolumn{2}{|c|}{$\begin{array}{c}\text { Effective } \\
\text { Temperature }\end{array}$} \\
\hline & New & Old & New & $\mathrm{Old}^{\mathrm{a}}$ \\
\hline J003957.00+410114.6 & M4 I & M0 I & 3650 & 3775 \\
\hline $\mathrm{J} 004035.08+404522.3$ & M2 I & M2.5 I & 3750 & 3775 \\
\hline J004047.82+410936.4 & M3 I & M3 I & 3650 & 3725 \\
\hline J004124.80+411634.7 & M2 I & $\mathrm{M} 3+? \mathrm{I}$ & 3725 & 3700 \\
\hline J004255.95+404857.5 & M2 I & M2 I & 3800 & 3750 \\
\hline J004428.71+420601.6 & M0 I & M0 I & 3825 & 3900 \\
\hline J004454.38+412441.6 & M0 I & M2 I & 3850 & 3800 \\
\hline J004514.95+414625.6 & M1 I & M2 I & 3800 & 3750 \\
\hline
\end{tabular}

Note.

a Adjusted by $+75 \mathrm{~K}$; see text.

In the past, our standard procedure (e.g., Levesque et al. 2005, 2006; Massey et al. 2009; Levesque \& Massey 2012) has been to use the model fitting to determine $E(B-V)$. However, our recent experiences with fiber spectrophotometry has been disappointing. Hectospec uses an atmospheric dispersion compensator, and a careful study by Fabricant et al. (2008) demonstrates that the flux calibration of Hectospec data is good to a few percent on extended sources. However, we have found that flux calibrating point sources in crowded fields is considerably less successful. In many cases the reddenings required to fit the fluxed spectrophotometry are clearly non-physical, i.e., requiring zero or negative $E(B-V)$. We have encountered similar problems in modeling Magellanic Clouds RSGs using 2 Degree Field fiber data from the Australian Astronomical Observatory in a separate project. We have thus decided to adopt the median $E(B-V)=0.3$ $\left(A_{V}=1.0\right)$ found by Massey et al. (2009) for their sample of M31 RSGs. This is somewhat larger than the average reddenings of OB stars (Massey et al. 2007b), which is consistent with what we find from a comparison to the reddenings of OBs and RSGs in the Milky Way (Levesque et al. 2005) and Magellanic Clouds (Levesque et al. 2006), and which is attributable to circumstellar reddening by dust (Massey et al. 2005).

To minimize the effect that $A_{V}$ is poorly determined individually for our stars, we have chosen to rely on near-IR
(NIR) $K$-band photometry, as $A_{K}$ is only about $10 \%$ of $A_{V}$, and the bolometric correction is small and fairly insensitive to the temperature. This also has the advantage of avoiding the typical $\sim 0.9$ mag variability seen in $V$ (Levesque et al. 2007); no such variability is seen at $K$, a point also emphasized by Massey et al. (2009). Of the 255 stars in our sample, 37 have targeted $K$-band photometry using the NIR photometer FLAMINGOS (Massey et al. 2009); for the rest, we adopt 2MASS (Cutri et al. 2003) values where available. We perform the same conversion to bolometric luminosity in the same manner as Massey et al. (2009):

$$
\begin{aligned}
K & =K_{s}+0.04 \\
K_{0} & =K-0.12 A_{V}=K-0.12 \\
M_{K} & =K_{0}-24.40 \\
M_{\text {bol }} & =M_{K}+\mathrm{BC}_{K},
\end{aligned}
$$

where $K$ is the "standard" (such as it is) photometric band (see, e.g., Carpenter 2001), $K_{s}$ is the observed 2MASS or FLAMINGOS photometry, $K_{0}$ is the de-reddened $K$-band magnitude, $M_{K}$ is the absolute $K$-band magnitude where we have assumed a true distance modulus of 24.40 (0.76 Mpc, van den Bergh 2000), $M_{\mathrm{bol}}$ is the bolometric magnitude, and $\mathrm{BC}_{K}$ is the bolometric correction in the $K$-band, a small but positive number (e.g., Bessell et al. 1998; Levesque et al. 2005). We determine $\mathrm{BC}_{K}$ from the effective temperature $T_{\text {eff }}$ following Massey et al. (2009) as follows:

$$
\mathrm{BC}_{K}=7.149-1.5924\left(\frac{T_{\mathrm{eff}}}{1000 \mathrm{~K}}\right)+0.10956\left(\frac{T_{\mathrm{eff}}}{1000 \mathrm{~K}}\right)^{2},
$$

where the relationship is derived from the MARCS models. Finally, of course,

$$
\log L / L_{\odot}=\left(M_{\mathrm{bol}}-4.75\right) /-2.5 .
$$

\subsection{Comparison with Evolutionary Tracks}

Having derived physical properties for our sample of RSGs, we compare their location in the HRD to that predicted by the evolutionary tracks in Figure 4. In making this comparison, we encounter a difficulty: so far, only solar (Ekström et al. 2012) and sub-solar (Georgy et al. 2013) versions of the new Geneva 


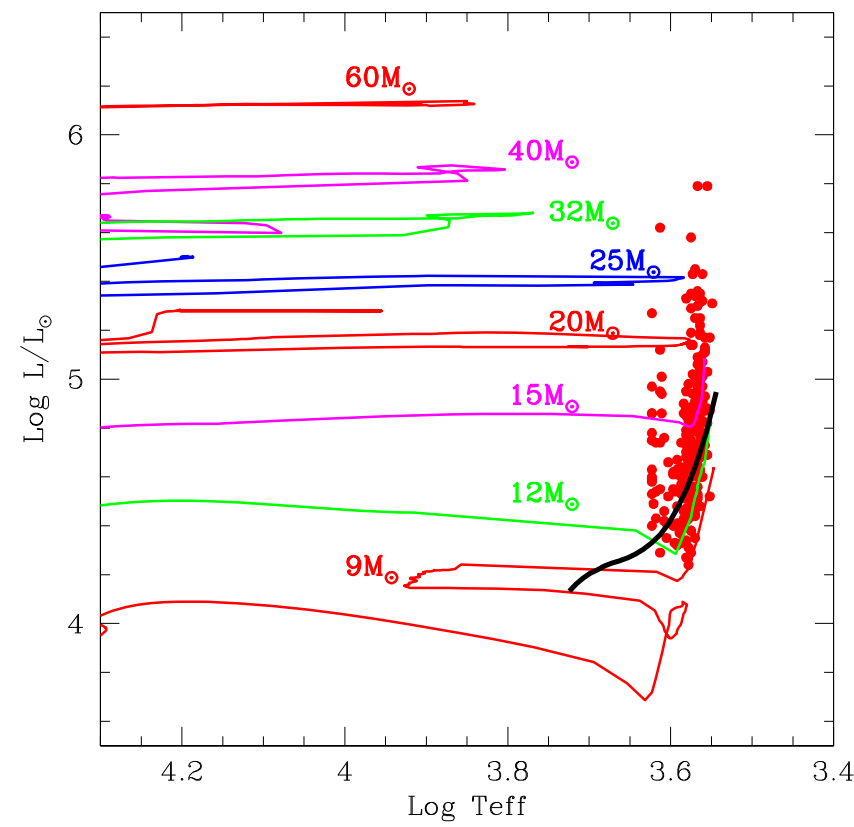

Figure 4. Location of the RSGs in the HRD. We have plotted the location of M31's RSGs in the HRD using the values in Table 2. The evolutionary tracks are from Ekström et al. (2012), computed for solar metallicity and an initial rotation of $40 \%$ of the critical breakup speed. The initial masses are shown for each track. The black curve corresponds to $V=20$, our estimated completeness limit.

tracks are available. Sanders et al. (2012) recently conducted a comprehensive study of the chemical abundances of M31's H II regions, finding that $\log (\mathrm{O} / \mathrm{H})+12=9.0-9.1(2 \times$ solar $)$ in the center of $\mathrm{M} 31$ with a shallow galactocentric gradient $\left(-0.01\right.$ to $\left.-0.02 \mathrm{dex} \mathrm{kpc}^{-1}\right)$, in accord with the older study of Zaritsky et al. (1994). (A value of $\log (\mathrm{O} / \mathrm{H})+12=8.7$ is considered typical of the solar neighborhood, and hence our description of $2 \times$ solar.) However, one of the most intriguing results to come out of the Sanders et al. (2012) study is the large scatter in the oxygen abundance measures, which these authors argue is intrinsic and not observational. As their Figure 8 shows, at a galactocentric distance of $10-12 \mathrm{kpc}$, the values for $\log (\mathrm{O} / \mathrm{H})+12$ vary from 8.5 to 9.3 dex. Thus, the solar metallicity evolutionary tracks are not inappropriate either. However, the changes in the physical parameters we would have derived using solar metallicity models are extremely modest. We would have to decrease our effective temperatures by $75 \mathrm{~K}$, the approximate systematic difference between the solar and $2 \times$ solar MARCS atmospheric models as discussed earlier. This translates to a very modest $\sim 0.01$ dex decrease in the effective temperature and a 0.02 dex decrease in the bolometric luminosity, i.e., the change is comparable to the point sizes in the figure.

The agreement between the evolutionary tracks and the locations of stars in the HRD is nothing short of remarkable. There are only four stars whose luminosities appear to be higher than what the tracks allow. The two highest luminosity stars in the figure are J004520.67+414717.3 (M1 I) and $\mathrm{J} 004539.99+415404.1$ (M3 I) are both found at $X / R>0.9$ where there $i s$ considerable overlap in the radial velocities of RSGs and foreground stars; we thus are relying primarily on their photometry to classify these as supergiant members. The photometry of the first of these clearly places it in the RSG category (J004520.67+414717.3 has $B-V=2.68$,
$V-R=1.55)$, while the photometry of the second is more marginal (J004539.99+415404.1, $B-V=1.70, V-R=$ 0.94). The next two most luminous stars are not necessarily discrepant with the evolutionary tracks, as somewhere between 25 and $32 M_{\odot}$ may extend over to the RSG phase. These stars have $\log L / L_{\odot} \sim 5.6$ and are J004428.12 $+415502.9(\mathrm{~K} 2 \mathrm{I}, X / R=0.75)$ and $\mathrm{J} 004428.48+415130.9$ (M1 I, $X / R=0.82)$, located where where there is more support from the radial velocities for membership. However, the photometry of J004428.12+415502.9 has an unusually large $B-V$ value (2.05) for its $V-R$ value (0.95). Finally, there are three stars all with $\log L / L_{\odot}$ of $5.45-5.47$, J004125.23+411208.9 (M0 I, $X / R=-0.29$ ), J004312.43 $+413747.1(\mathrm{M} 2 \mathrm{I}, X / R=0.45)$, and J004514.91+413735.0 (M1 I, $X / R=0.64$ ), where the radial velocities leave no question as to membership; these stars, however, are consistent with the $25 M_{\odot}$ track. We conclude that only J004520.67+414717.3 seems to be discrepant with what the evolutionary tracks predict; this high luminosity star may warrant further investigation.

There is an interesting bifurcation in the location of the RSGs in the HRD, with two branches separated by increasingly large differences in effective temperatures at higher luminosities. Is this real, or an artifact of our analysis? The warmer stars are those classified as early K-type, while the cooler stars are classified as M-type; the larger gap at higher luminosities is a reflection that the median spectral type for the M-type stars increases with luminosity. For instance, for the stars with $\log L / L_{\odot}$ less than 5.0 , the median spectral type is M0 I; for stars with $\log L / L_{\odot} \geqslant 5.0$, the median spectral type is M2 I. (Correspondingly the median effective temperatures are 3850 $\mathrm{K}$ and $3750 \mathrm{~K}$, respectively.) This bifurcation is partially reflected in the evolutionary tracks; we see for the $25 M_{\odot}$ track a short loop that results in increased time spent in region occupied by the early K-type supergiants. To quantify this further, in Table 4 we compute the amount of time the evolutionary tracks predict a star will spend in the RSG regime $(<4300 \mathrm{~K})$ as a function of effective temperature, in the effective temperature range $4100-4350 \mathrm{~K}$ and $3600-4100 \mathrm{~K}$. We see that the gap (which occurs around 4100-4150 K) actually occurs in the $25 M_{\odot}$ track. Although the gap is not present in the 15 or $20 M_{\odot}$ track we note that the percentage of "warm" versus "cool" RSGs does increase with increasing luminosity, in much the same way as is reflected by the quantity of stars seen in the HRD. A more exact comparison will be made when the higher metallicity tracks become available.

The numbers in Table 4 do reveal that the $25 M_{\odot}$ track may not extend quite far enough to cooler temperatures, although this may well be an artifact of the metallicity of the solar track being too low for the M31 RSGs; we expect the Hayashi limit ${ }^{9}$ to shift to cooler temperatures with increasing metallicities (see the discussion in Levesque et al. 2006).

\section{SUMMARY AND DISCUSSION}

We have shown that the photometric separation of RSGs and red foreground stars using a $B-V$ versus $V-R$ diagram works extremely well, at least at the $1.5 \times$ solar metallicity of M31. The exceptions were stars whose photometry was

\footnotetext{
9 The Hayashi limit is the coolest temperature at which a star would remain hydrostatically stable (Hayashi \& Hoshi 1961).
} 
Table 4

Lifetimes (years) for RSGs from the Ekström et al. (2012) Evolutionary Tracks

\begin{tabular}{lccccccc}
\hline \hline Initial Mass & \multicolumn{5}{c}{ Effective Temperatures $(\mathrm{K})$} & \\
\cline { 2 - 7 }$\left(M_{\odot}\right)$ & $3700-3800$ & $3800-3900$ & $3900-4000$ & $4000-4100$ & $4100-4200$ & $4200-4300$ \\
\hline 25 & 0 & 63,000 & 30,00 & 800 & 3000 & 12,000 \\
20 & 151,000 & 149,000 & 20,000 & 6000 & 4300 & 5400 & $15 \%$ \\
15 & 380,000 & 8000 & 3000 & 2000 & 2000 & 2000 & $0.3 \%$ \\
\hline
\end{tabular}

Note.

a "Warm" is $4100-4300 \mathrm{~K}$ while "cool" is $<4100 \mathrm{~K}$.

suspect, or marginally on the borderline between that expected for the two sequences. There was only one exception out of 354 stars.

The agreement between the evolutionary tracks and the locations of RSGs in the HRD is excellent; there is one star, J004520.67+414717.3, whose photometry places it clearly in the RSG category and yet its high luminosity $\left(\log L / L_{\odot} \sim 5.8\right)$ appears to be inconsistent with what the evolutionary tracks predict; it will be interesting to see if this star is still discrepant when higher metallicity tracks become available. The bifurcation in the effective temperatures that increases with increasing luminosities is only partially reflected in the evolutionary tracks. This is consistent with the small extra loop in the $25 M_{\odot}$ track but is not indicated by the other tracks. At higher luminosities the median spectral type becomes slightly later than at lower luminosities; i.e., higher mass RSGs spend more time at cooler temperatures than do stars of lower mass.

\subsection{Completeness of the Current Sample}

Using the same LGGS photometry that we employ here, Massey et al. (2009) identified 437 stars as likely RSGs based on the two-color diagrams. Of these, we have new spectra and spectral types for 255 (about 60\%) of these stars. The Massey et al. (2009) study determined radial velocities for 124 of the 437 stars in the original sample. We have new observations of 74 of those 124 stars, and have included the 50 additional stars with radial velocities from Massey et al. (2009) in Figure 2. We successfully compute physical properties for 192 stars from these 255 stars; the others are either lacking 2MASS $K$-band photometry or we were unable to fit their effective temperatures. Massey et al. (2009) only measured physical properties for 16 stars (their radial velocity spectra were at high dispersion and did not provide the necessary amount of wavelength coverage); we have new observations for right of those stars here, and have compared the derived properties in Table 3. We have chosen to ignore the other eight stars, as Massey et al. (2009) inadvertently used solar (rather than $2 \times$ solar) models in deriving their physical properties. Thus Figure 4 and Table 2 reflect the physical properties of about $44 \%$ of the original sample.

\subsection{Extension to Fainter Limits}

As discussed in the Introduction, an exciting possibility is to use the luminosity function of RSGs in M31 to provide a constraint on the mass-loss rates of these stars. In his preliminary work, C. Georgy (2015, private communication) has shown that the slope of the luminosity function is fairly sensitive to the assumed mass-loss rates. However, the slopes of the luminosity functions are fairly parallel for $\log L / L_{\odot}>4.5$; for this to be useful requires extending our knowledge of the RSG populations down to $\log L / L_{\odot} \sim 4.2$ or lower.

We include in Figure 4 the current completeness limit set by $V \leqslant 20.0$ as a solid black line. ${ }^{10}$ Including the coolest stars, we would consider the current sample complete to $\log L / L_{\odot}$ of 4.8 . Thus, we would need to extend this work about 0.6 dex fainter, or about $1.5 \mathrm{mag}$, to $V \sim 21.5$. The photometry in the LGGS is good enough to distinguish foreground and RSGs at that level: the expected $1 \sigma$ error in $B-V$ would be about 0.05 (c.f. Table 6 in Massey et al. 2006) which is still acceptable (see Figure 1). What is lacking, however, is the necessary NIR photometry. The 2MASS point-source catalog peters out around $K_{s} \sim 15.0$ in M31; optimally we would need to extend this to $K_{s} \sim 16.5$. It may not be practical to obtain optical spectroscopy at this level; Massey et al. (2009) notes that extending the study to just $V=21.0$ would increase the number of RSG candidates to 1750 ; we calculate here that extension to $V=21.5$ would then include $\sim 3200$ stars.

Photometry alone would allow us to fix the effective temperatures well enough to determine the bolometric luminosities. According to the MARCS models, $\Delta T_{\text {eff }} / \Delta(J-K)=$ $-1777 \mathrm{~K} \mathrm{mag}^{-1}$ and $\Delta \mathrm{BC}_{K} / \Delta(J-K)=1.33$, both extremely linear ( $\mathrm{rms}$ of $10 \mathrm{~K}$ and $0.005 \mathrm{mag}$, respectively) over the RSG effective temperature range. Thus a signal-to-noise ratio of 30 at $J$ and $K_{S}$ would allow us to determine a $\log L / L_{\odot}$ of 0.02 dex (about the size of the points in Figure 4), and provide a temperature precision of $70 \mathrm{~K}$, just a little worse than the $50 \mathrm{~K}$ precision we claim for the spectral fitting. We have proposed to do exactly this in the next observing season.

\subsection{Concluding Remarks}

Meynet et al. (2015) has emphasized the importance of mass loss during the RSG phase regarding the further evolution of these stars, and has raised the alarming possibility that the majority of blue and yellow supergiants we observe are postRSG objects. Modern estimates of the mass-loss rates $\dot{M}$ are of the order $10^{-4} M_{\odot} \mathrm{yr}^{-1}$ (van Loon et al. 2005), but the total amount of mass lost during the RSG phase as a function of luminosity is poorly constrained observationally for several reasons. First, it requires measuring mid-IR excesses for RSGs with known distances; this sample contains only about $\sim 20$ RSGs (see, e.g., Table 3 in van Loon et al. 2005). Second, even so these measurements only tell us the dust production rate; we then have to apply an uncertain dust-to-gas ratio (300-500?). Third, and most importantly, knowing the mass-loss rates for a scant number of stars does not tell us much about what the time-averaged values actually are, as most of the mass lost

\footnotetext{
${ }^{10}$ There are stars below this line owing to the fact that we used $K$-band photometry to construct the HRD, and that the $V$-band brightness of RSGs is variable at the $0.9 \mathrm{mag}$ level or beyond (Levesque et al. 2007).
} 
during the RSG phase happens during relatively brief outbursts. The star VY CMa may be the most spectacular example, as its circumstellar material suggests that its mass-loss rate was a factor of 20 higher in the past than it is at present (Smith et al. 2001; Decin et al. 2006).

Extension of our work here to fainter limits now has the potential of allowing us to measure the time-average mass-loss rates using the luminosity functions of these objects. We have established that our photometric technique is sufficient to distinguish RSGs from foreground stars, and combining this technique with NIR photometry has the potential for addressing this in a novel way, as outlined by Meynet et al. (2015) and Georgy et al. (2015). A more complete knowledge of the RSG population of M31 should allow us to answer this intriguing question.

We are grateful to the Steward Observatory Time Allocation Committee for their generous allocation of observing time on the MMT, and to Perry Berlind, Mike Calkins, and Marc Lacasse for their excellent support of Hectospec. P.M. would particularly like to thank Calkins for organizing and graciously hosting a very pleasant Thanksgiving dinner for all of the Mt Hopkins observers during the November observing run. Nelson Caldwell managed the difficult task of queue scheduling our program. Collaborator Kathryn Neugent participated in these observations and encouraged devoting the "spare" Hectospec fibers to this additional project; she was also kind enough to make useful comments on a draft of the manuscript, as did Cyril Georgy, Georges Meynet, and Sylvia Esktröm. An anonymous referee provided constructive comments. Emily Levesque and Joe Llama provided useful help with some of the coding for fitting the MARCS models. This publication makes use of data products from the Two Micron All Sky Survey, which is a joint project of the University of Massachusetts and the Infrared Processing and Analysis Center/California Institute of Technology, funded by the National Aeronautics and Space Administration and the National Science Foundation (NSF). K.A.E.'s work was supported through the NSF's Research Experiences for Undergraduates program through Northern Arizona University and Lowell Observatory (AST1461200), and P.M. was partially supported by the NSF through AST-1008020 and by Lowell Observatory.

Facility: MMT (Hectospec).

\section{REFERENCES}

Bessell, M. S., Castelli, F., \& Plez, B. 1998, A\&A, 333, 231

Carpenter, J. M. 2001, AJ, 121, 2851

Cutri, R. M., Skrutskie, M. F., van Dyk, S., et al. 2003, yCat, 2246, 0

Decin, L., Hony, S., de Koter, A., et al. 2006, A\&A, 456, 549

Drout, M. R., Massey, P., Meynet, G., Tokarz, S., \& Caldwell, N. 2009, ApJ, 703, 441

Ekström, S., Georgy, C., Eggenberger, P., et al. 2012, A\&A, 537, A146

Evans, K. A., \& Massey, P. 2015, AJ, 150, 149

Fabricant, D., Fata, R., Roll, J., et al. 2005, PASP, 117, 1411

Fabricant, D. G., Kurtz, M. J., Geller, M. J., et al. 2008, PASP, 120, 1222

Fujii, M. S., \& Portegies Zwart, S. 2011, Sci, 334, 1380

Georgy, C., Ekström, S., Eggenberger, P., et al. 2013, A\&A, 558, A103

Georgy, C., Ekström, S., Hirschi, R., et al. 2015, IAUGA, 22, 2256831

Gies, D. R., \& Bolton, C. T. 1986, ApJS, 61, 419

Hayashi, C., \& Hoshi, R. 1961, PASJ, 13, 442

Hurley-Keller, D., Morrison, H. L., Harding, P., \& Jacoby, G. H. 2004, ApJ, 616,804

Kippenhahn, R., \& Weigert, A. 1990, Stellar Structure and Evolution (Berlin: Springer)

Levesque, E. M., \& Massey, P. 2012, AJ, 144, 2

Levesque, E. M., Massey, P., Olsen, K. A. G., et al. 2005, ApJ, 628, 973

Levesque, E. M., Massey, P., Olsen, K. A. G., et al. 2006, ApJ, 645, 1102

Levesque, E. M., Massey, P., Olsen, K. A. G., \& Plez, B. 2007, ApJ, 667, 202

Maeder, A., Lequeux, J., \& Azzopardi, M. 1980, A\&A, 90, L17

Massey, P. 1998, ApJ, 501, 153

Massey, P. 2006, ApJL, 638, L93

Massey, P. 2013, NewAR, 57, 14

Massey, P., McNeill, R. T., Olsen, K. A. G., et al. 2007a, AJ, 134, 2474

Massey, P., Neugent, K., \& Smart, B. 2016, AJ, in press

Massey, P., Olsen, K. A. G., Hodge, P. W., et al. 2006, AJ, 131, 2478

Massey, P., Olsen, K. A. G., Hodge, P. W., et al. 2007b, AJ, 133, 2393

Massey, P., Plez, B., Levesque, E. M., et al. 2005, ApJ, 634, 1286

Massey, P., Silva, D. R., Levesque, E. M., et al. 2009, ApJ, 703, 420

Meynet, G., Chomienne, V., Ekström, S., et al. 2015, A\&A, 575, A60

Neugent, K. F., \& Massey, P. 2014, ApJ, 789, 10

Neugent, K. F., Massey, P., \& Georgy, C. 2012a, ApJ, 759, 11

Neugent, K. F., Massey, P., Skiff, B., et al. 2010, ApJ, 719, 1784

Neugent, K. F., Massey, P., Skiff, B., \& Meynet, G. 2012b, ApJ, 749, 177

Sanders, N. E., Caldwell, N., McDowell, J., \& Harding, P. 2012, ApJ, 758, 133

Schlegel, D. J., Finkbeiner, D. P., \& Davis, M. 1998, ApJ, 500, 525

Smartt, S. J., Eldridge, J. J., Crockett, R. M., \& Maund, J. R. 2009, MNRAS, 395, 1409

Smith, N., Humphreys, R. M., Davidson, K., et al. 2001, AJ, 121, 1111

Sofue, Y., \& Kato, T. 1981, PASJ, 33, 449

Tonry, J., \& Davis, M. 1979, AJ, 84, 1511

van den Bergh, S. 2000, The Galaxies of the Local Group (Cambridge: Cambridge Univ. Press)

van Loon, J. T., Cioni, M.-R. L., Zijlstra, A. A., \& Loup, C. 2005, A\&A, 438, 273

Zaritsky, D., Kennicutt, R. C., Jr., \& Huchra, J. P. 1994, ApJ, 420, 87 